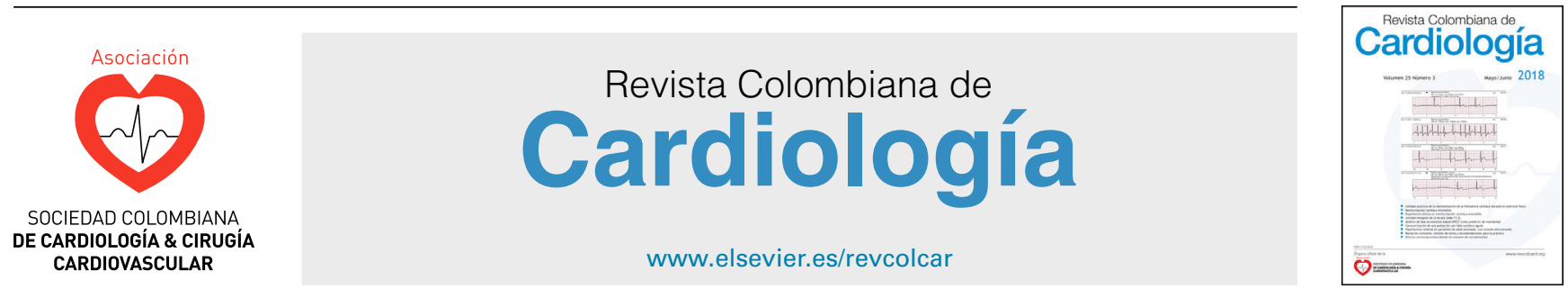

CARDIOLOGÍA DEL ADULTO - REVISIÓN DE TEMAS

\title{
Miocarditis por gram negativos
}

\section{María Pérez ${ }^{\mathrm{a}, *}$, Iván Noreña ${ }^{\mathrm{b}}$, Carlos Ortiz $^{\mathrm{c}}$, Rey Dairo ${ }^{\mathrm{c}}$, Henry Millán ${ }^{\mathrm{b}}$, Guillermo Sánchez ${ }^{\mathrm{b}}$, Fultón Perea ${ }^{\mathrm{a}}$, Laura Rincón $^{\mathrm{d}}$, Silvia Rincón ${ }^{\mathrm{d}}$ y Julián Alberto Naranjo ${ }^{\mathrm{e}}$}

\author{
a Departamento de Medicina Interna, Fundación Cardioinfantil-Instituto de Cardiología, Bogotá, Colombia \\ b Departamento de Infectología, Fundación Cardioinfantil-Instituto de Cardiología, Bogotá, Colombia \\ c Departamento de Cardiología, Fundación Cardioinfantil-Instituto de Cardiología, Bogotá, Colombia \\ d Medicina, Universidad Industrial de Santander, Bucaramanga, Colombia \\ e Fundación Cardioinfantil-Instituto de Cardiología, Bogotá, Colombia
}

Recibido el 4 de octubre de 2019; aceptado el 28 de mayo de 2020

Disponible en Internet el 29 de octubre de 2020

\author{
PALABRAS CLAVE \\ Miocarditis; \\ Enterobacteriaceae; \\ Salmonella; \\ Shigella; \\ Vibrio; \\ Yersinia; \\ Campylobacter; \\ Clostridium
}

\begin{abstract}
Resumen
Objetivo: describir las características epidemiológicas, clínicas y microbiológicas de los pacientes que cursan con miocarditis por Enterobacterias.

Métodos: se realizó una revisión sistemática de la literatura, en la que se incluyeron Pubmed, Ovid, Scopus, SciELO y LILACS sin exclusión por tipo de idioma. La población objetivo de estudio fueron los pacientes con diagnóstico de infección bacteriana por bacilo gram negativo mediante cultivo, técnicas moleculares o histopatología, y quienes presentaban biopsia de miocardio o, en su defecto, resonancia magnética cardiaca con hallazgos sugestivos de miocarditis.

Resultados: se encontraron 742 artículos, de los cuales se incluyeron 24; en estos se reportaron 27 pacientes. La edad promedio fue de 31 años. El 81\% de los pacientes eran de sexo masculino. El síntoma principal fue diarrea (80\%), seguido de fiebre $(53 \%)$ y dolor torácico (38\%). El $37 \%$ de los pacientes fallecieron. El hallazgo más común en el electrocardiograma fue la elevación del segmento ST $(36,7 \%)$. En quienes se realizó ecocardiograma se encontraron anormalidades en $50 \%$ de los casos, siendo más frecuente la disminución en la fracción de eyección.

El microorganismo más común fue el Campylobacter jejuni, seguido por Salmonela sp.

Conclusiones: la miocarditis causada por enterobacterias es más frecuente en pacientes adultos jóvenes de sexo masculino. Los síntomas gatrointestinales suelen estar presentes al momento de la presentación clínica. El diagnóstico requiere de alta sospecha clínica teniendo en cuenta que las anormalidades eléctricas y en ecocardiograma no se encuentran en todos los pacientes. (c) 2020 Sociedad Colombiana de Cardiología y Cirugía Cardiovascular. Publicado por Elsevier España, S.L.U. Este es un artículo Open Access bajo la licencia CC BY-NC-ND (http:// creativecommons.org/licenses/by-nc-nd/4.0/).
\end{abstract}

\footnotetext{
* Autor para correspondencia.

Correo electrónico: mariaperezardila@gmail.com (M. Pérez).
} 


\section{KEYWORDS}

Myocarditis;

Enterobacteriaceae;

Salmonella;

Shigella;

Vibrio;

Yersinia;

Campylobacter;

Clostridium

\section{Gram-Negative Myocarditis}

\begin{abstract}
Objective: To describe the epidemiological, clinical, and microbiological characteristics of patients with myocarditis due to Enterobacteria.

Methods: A systematic review was carried out on the literature, which included Pubmed, Ovid, Scopus, SciELO, and LILACS, with no exclusions due to language. The target population of the study were patients with a diagnosis of bacterial infection due to gram negative bacillus by means of a culture, or using molecular or histopathology technique. They also had to have had a myocardial biopsy or, if not, a cardiac magnetic resonance scan with findings suggestive of myocarditis.

Results: Out of a total of 742 articles found, 24 of these, in which 27 patients were described, were included. The mean age was 31 years, and $81 \%$ were male. The main symptom was diarrhoea $(80 \%)$, followed by fever $(53 \%)$, and chest pain $(38 \%)$. More than one-third $(37 \%)$ of the patients died. The most common finding on the electrocardiogram (ECG) was elevation of the ST segment $(36.7 \%)$. Abnormalities were found in $50 \%$ of the cases, on whom a cardiac ultrasound was performed, with a decrease in the ejection fraction being the most common.

The most common microorganism was Campylobacter jejuni, followed by Salmonella spp. Conclusions: Myocarditis caused by enterobacteria is most common in young male patients. The gastrointestinal symptoms are usually present from the clinical onset. The diagnosis requires a high clinical suspicion, taking into account that the abnormalities in the ECG and cardiac ultrasound are not found in all patients.

(c) 2020 Sociedad Colombiana de Cardiología y Cirugía Cardiovascular. Published by Elsevier España, S.L.U. This is an open access article under the CC BY-NC-ND license (http:// creativecommons.org/licenses/by-nc-nd/4.0/).
\end{abstract}

\section{Introducción}

Miocarditis se define como el proceso inflamatorio del miocardio el cual resulta en daño de los miocitos. El diagnóstico incluye criterios histológicos e inmunohistoquímica ${ }^{1}$. Es el diagnóstico final de las admisiones por razones cardiovasculares en $0.5 \%$ de los casos, pero puede encontrarse en el 10 a $17 \%$ de los pacientes con miocardiopatía no explicada quienes son llevados a biopsia endomiocárdica ${ }^{2}$. En Colombia se desconoce la prevalencia de esta patología.

La etiología puede estar dada por infecciones, enfermedades autoinmunes, reacciones de hipersensibilidad, fármacos o agentes físicos ${ }^{1}$. La principal causa son las infecciones virales, incluso cuando no se detectan virus en pruebas serológicas ${ }^{3}$.

Las pruebas iniciales para el diagnóstico de miocarditis son el electrocardiograma, los biomarcadores y el ecocardiograma; con base en los resultados es posible establecer el riesgo de miocarditis y determinar si el paciente requiere biopsia endomiocardica ${ }^{1}$.

La mayoría de los individuos con miocarditis permanecen asintomáticos o la enfermedad se resuelve espontáneamente; sin embargo, un 10 a un $20 \%$ pueden desarrollar miocardiopatía dilatada, y de esta población aproximadamente la mitad requerirá trasplante cardíaco ${ }^{4,5}$.

\section{Marco teórico y estado del arte}

El proceso inflamatorio en la miocarditis requiere la mediación de células del sistema inmune tanto innato como adaptativo ${ }^{4}$. En el caso de las infecciones, los microorganismos que infectan el órgano pueden desencadenar la respuesta inmune que facilita la liberación de antígenos intracelulares, que son reconocidos por el sistema inmune como externos. Las reacciones cruzadas también pueden estar asociadas a la degeneración en el reconocimiento por parte del complejo mayor de histocompatibilidad ${ }^{4}$. Este mecanismo no solo se ha implicado en la respuesta a infecciones virales, sino también a infecciones por otro tipo de microorganismos incluyendo las bacterias ${ }^{9}$.

La miocarditis bacteriana puede desarrollarse por invasión directa de las bacterias o por toxinas elaboradas por el patógeno, como en el caso de infección por Corynebacterium diphtheriae y Clostridium $s p$. Las causas de disfunción ventricular incluyen inflamación miocárdica y necrosis, disfunción ventricular inducida por toxinas, disfunción miocárdica inducida por sepsis, y remodelamiento ventricular ${ }^{6}$

La verdadera prevalencia de la miocarditis por causas bacterianas es difícil de estimar. Estudios posmortem han reportado una prevalencia de 0.2 a $1.5 \%{ }^{7}$. La presentación clínica depende del foco infeccioso inicial y de los signos de disfunción miocárdica. El grado de compromiso cardíaco varía de acuerdo con la etiología y la severidad del proceso infeccioso ${ }^{6}$.

Los hallazgos histopatológicos más comunes en la miocarditis bacteriana son los exudados neutrofílicos en la fase aguda y los infiltrados crónicos con formación de abscesos y necrosis de miocitos. En la miocarditis inducida por toxinas existe un predominio linfohistiocítico ${ }^{8}$. 
Tabla 1 Diagnósticos clínicos para diagnóstico de miocarditis

Presentación clínica

- Dolor torácico agudo, pericárdico o pseudoisquémico

- Nuevo inicio (días a 3 meses) o empeoramiento de disnea en ejercicio o reposo o fatiga, con o sin signos de falla cardíaca

- Inicio crónico/subagudo (> 3 meses) o empeoramiento de disnea en ejercicio o reposo o fatiga, con o sin signos de falla

cardíaca

- Palpitaciones o síntomas de arritmia inexplicados, síncope o muerte súbita abortada

- Shock cardiogénico inexplicado

Criterios diagnósticos

1 EKG/Holter/prueba de estrés

Resultados anormales en EKG, Holter o prueba de estrés con cualquiera de los siguientes: bloqueo de I a III grado, cambios en segmento/onda ST/T, pausa sinusal, taquicardia ventricular o fibrilación auricular, reducción altura onda R, retraso en conducción intraventricular, ondas Q, bajo voltaje, latidos prematuros, taquicardia supraventricular

2 Marcadores elevados: TnT, Tnl

3 Anormalidades estructurales o funcionales en imagen cardíaca (ECO, angio, RM)

Anormalidades estructurales o funcionales de novo en ventrículo izquierdo o derecho: alteraciones en motilidad de pared, anormalidades en función diastólica o función sistólica global, con o sin dilatación ventricular, con o sin aumento de grosor de ventrículo, con o sin efusión pericárdica, con o sin trombos intracavitarios.

4 Caracterización de tejido por RM

Edema o LGE de patrón miocárdico clásico

Sospecha clínica de miocarditis si: > 1 criterio diagnóstico clínico y más de 1 criterio de las diferentes categorías en ausencia de enfermedad coronaria significativa (> 50\% de estenosis), o de condición cardiovascular preexistente o enfermedad extra cardíaca que pueda explicar el síndrome

En ausencia de la realización de biopsia, la diferenciación entre sepsis inducida por depresión miocárdica y miocarditis bacteriana puede ser difícil ya que ambas entidades producen disfunción miocárdica y elevación de biomarcadores $^{6}$; en estos casos la resonancia magnética provee información sobre aspectos funcionales y morfológicos del miocardio tanto de valor pronóstico como diagnóstico ${ }^{1}$. Para el diagnóstico mediante resonancia magnética uno de los abordajes incluye los criterios de Lake Louise, que consiste en secuencias de realce tardío, además del hallazgo de edema en secuencias T2 y T1 antes de la administración de contraste ${ }^{9,10}$. La resonancia magnética cuenta con sensibilidad y especificidad tan alta como del 100 y del $90 \%$ al ser comparada con estudios histológicos ${ }^{11}$. El patrón de distribución también permite establecer la etiología de la miocarditis $^{12}$.

Por lo anterior, la resonancia magnética puede ser un suplemento, o incluso reemplazar la biopsia, en pacientes con miocarditis clasificados como de riesgo bajo o intermedio (dolor torácico, anormalidades eléctricas que no amenazan la vida y que presentan disfunción ventricular izquierda leve a moderada o alteraciones en la contractilidad). Sin embargo, en pacientes de alto riesgo (falla cardíaca, descompensada, arritmias que amenazan la vida o disfunción ventricular izquierda severa) debe considerarse la realización de la biopsia ${ }^{1,13}$. Dada la no disponibilidad global de esta prueba, se han propuesto criterios clínicos y de imágenes para realizar el diagnóstico (tabla 1$)^{1}$.

\section{Caso}

Paciente de sexo masculino, de 49 años de edad, con antecedente de hipertensión arterial e hipotiroidismo, quien consultó por 3 días de evolución con deposiciones líquidas en cantidad de 15 a 20 al día sin sangre, sin moco y sin fiebre; el último día del cuadro clínico se asoció dolor abdominal tipo cólico leve. Al ingreso a urgencias la tensión arterial era de $118 / 70 \mathrm{~mm} \mathrm{Hg}$, se encontró taquicárdico, sin taquipnea, afebril y sin dolor a la palpación abdominal (tabla 2).

El hemograma no mostraba leucocitosis ni neutrofilia, ni alteración en el valor de hemoglobina o plaquetas. El coproscópico era de características inflamatorias, por lo cual se solicitó panel gastrointestinal múltiple (FilmArray) en el que identificó Salmonella spp y Escherichia coli enteropatogénica (tabla 3).

Durante su permanencia en urgencias tuvo un episodio de dolor precordial opresivo de intensidad 9/10 durante 40 minutos, asociado a diaforesis profusa, sin disnea y sin palpitaciones. El electrocardiograma mostraba onda $\mathrm{T}$ invertida en cara inferior sin otros hallazgos, y la troponina inicial estaba elevada (valor $18.2 \mathrm{ng} / \mathrm{ml}$, valor normal hasta 0.026 $\mathrm{ng} / \mathrm{ml}$ ), por lo que se realizó diagnóstico de infarto agudo de miocardio. Se llevó a arteriografía, la cual mostró obstrucción del $40 \%$ de la coronaria derecha. La función residual de flujo fue de 0.95 .

Por el dolor torácico asociado a elevación de troponina en el escenario de infección gastrointestinal, se realizó inicialmente un ecocardiograma en el cual se encontró remodelación concéntrica del ventrículo izquierdo, FEVI del $60 \%$ y disfunción diastólica tipo I, sin otras alteraciones. Adicionalmente, se realizó resonancia magnética cardíaca para estudio de miocarditis. En las imágenes se observó edema miocárdico, realce tardío en relación a la zona de edema, que indica cicatriz en localización subepicárdica, con volumen y función sistólica biventricular conservada (fig. 1).

El paciente recibió en total 14 días de manejo con ciprofloxacina. Tanto el coprocultivo como los hemocultivos fueron negativos. 


\begin{tabular}{|c|c|c|c|c|c|c|c|c|c|c|}
\hline No & Autor & Año & Edad & Sexo & $\begin{array}{l}\text { Método } \\
\text { diagnóstico }\end{array}$ & Microorganismo & Síntomas & Alteraciones en el EKG & Biomarcador & Muerte \\
\hline 1 & $\begin{array}{l}\text { Hennigar } \\
\text { et al. } .^{21}\end{array}$ & 1953 & 2 & $M$ & Aglutinación & $\begin{array}{l}\text { Salmonella no } \\
\text { typhi }\end{array}$ & $\begin{array}{l}\text { Fiebre, diarrea, } \\
\text { vómito, anorexia, } \\
\text { hepatomegalia y } \\
\text { esplenomegalia, } \\
\text { adenopatías }\end{array}$ & No reporte & No reporte & Sí \\
\hline 2 & $\begin{array}{l}\text { Rosenberg } \\
\text { et al. } 22\end{array}$ & 1964 & 3 & $\mathrm{~F}$ & Biopsia & $\begin{array}{l}\text { Klebsiella } \\
\text { pneumoniae }\end{array}$ & No reporte & No reporte & NE & Sí \\
\hline 3 & $\begin{array}{l}\text { Sanders } \\
\text { et al. }{ }^{23}\end{array}$ & 1964 & 62 & $\mathrm{~F}$ & Hemocultivos & $\begin{array}{l}\text { Salmonella } \\
\text { choleraesuis }\end{array}$ & $\begin{array}{l}\text { Disnea, dolor } \\
\text { torácico, anorexia, } \\
\text { diarrea }\end{array}$ & Aplanamiento de ondas $\mathrm{T}$ & No reporte & Sí \\
\hline 4 & $\begin{array}{l}\text { Langaker } \\
\text { et al. }{ }^{24}\end{array}$ & 1973 & 76 & M & $\begin{array}{l}\text { Hemocultivos } \\
- \\
\text { coprocultivo }\end{array}$ & $\begin{array}{l}\text { Salmonella } \\
\text { typhimurium }\end{array}$ & $\begin{array}{l}\text { Diarrea, vértigo, } \\
\text { confusión }\end{array}$ & $\begin{array}{l}\text { Ondas T negativas } \\
\text { precordiales. Disociación } \\
\text { AV }\end{array}$ & No reporte & Sí \\
\hline 5 & $\begin{array}{l}\text { Simonsen } \\
\text { et al. }\end{array}$ & 1980 & 24 & $M$ & $\begin{array}{l}\text { Contenido } \\
\text { colónico } \\
\text { autopsia }\end{array}$ & $\begin{array}{l}\text { Salmonella } \\
\text { typhimurium }\end{array}$ & Diarrea, náuseas & No reporte & No reporte & Sí \\
\hline 6 & Götz et al. ${ }^{26}$ & 1983 & 53 & M & Hemocultivo & $\begin{array}{l}\text { Salmonella } \\
\text { typhi murium }\end{array}$ & $\begin{array}{l}\text { Fiebre, dolor } \\
\text { abdominal }\end{array}$ & $\begin{array}{l}\text { Disminución voltaje, } \\
\text { onda T aplanada }\end{array}$ & NE & Sí \\
\hline 7 & Burt et al..$^{27}$ & 1990 & 29 & M & Hemocultivos & $\begin{array}{l}\text { Salmonella } \\
\text { heidelberg }\end{array}$ & $\begin{array}{l}\text { Diarrea, dolor } \\
\text { abdominal, muerte } \\
\text { súbita }\end{array}$ & Fibrilación ventricular & No reporte & Sí \\
\hline 8 & $\begin{array}{l}\text { Rubesteins } \\
\text { JS et al. }{ }^{28}\end{array}$ & 1993 & 8 & $\mathrm{~F}$ & Coprocultivo & Shigella sonnei & $\begin{array}{l}\text { Letargia, cefalea, } \\
\text { fiebre, emesis y } \\
\text { diarrea }\end{array}$ & $\begin{array}{l}\text { Ectopia ventricular } \\
\text { prematura con } \\
\text { disminución del voltaje }\end{array}$ & NE & No \\
\hline 9 & $\begin{array}{l}\text { Rubesteins } \\
\text { JS et al. }{ }^{28}\end{array}$ & 1993 & 10 & $\mathrm{~F}$ & $\begin{array}{l}\text { Cultivo de } \\
\text { aspirado } \\
\text { gástrico }\end{array}$ & Shigella sonnei & $\begin{array}{l}\text { Diarrea, cefalea, } \\
\text { fiebre }\end{array}$ & $\begin{array}{l}\text { Bloqueo de segundo } \\
\text { grado intermitente }\end{array}$ & $\mathrm{NE}$ & No \\
\hline 10 & $\begin{array}{l}\text { Leon F } \\
\text { et al. }{ }^{29}\end{array}$ & 1997 & 59 & $M$ & Coprológico & Vibrio cholera & $\begin{array}{l}\text { Diarrea, náuseas, } \\
\text { vómito }\end{array}$ & $\begin{array}{l}\text { Bloqueo trifascicular: } \\
\text { Bloqueo AV primer } \\
\text { grado, bloqueo completo } \\
\text { rama derecha, } \\
\text { hemibloqueo postero } \\
\text { inferior izquierdo }\end{array}$ & CPK elevada & No \\
\hline
\end{tabular}




\begin{tabular}{|c|c|c|c|c|c|c|c|c|c|c|}
\hline No & Autor & Año & Edad & Sexo & $\begin{array}{l}\text { Método } \\
\text { diagnóstico }\end{array}$ & Microorganismo & Síntomas & Alteraciones en el EKG & Biomarcador & Muerte \\
\hline 11 & $\begin{array}{l}\text { Johnson JS } \\
\text { et al. } .^{30}\end{array}$ & 1998 & 83 & M & Urocultivo & $\begin{array}{l}\text { Serratia } \\
\text { marcescens }\end{array}$ & Disnea, ortopnea & NE & $\mathrm{NE}$ & $\mathrm{Si}$ \\
\hline 12 & $\begin{array}{l}\text { Neuwirth C, } \\
\text { et al. }{ }^{31}\end{array}$ & 1999 & 1 mes & M & $\begin{array}{l}\text { Biopsia de } \\
\text { miocardio }\end{array}$ & $\begin{array}{l}\text { Salmonella } \\
\text { virchow }\end{array}$ & Fiebre, muerte súbita & $\mathrm{NE}$ & NE & $\mathrm{Si}$ \\
\hline 13 & $\begin{array}{l}\text { Cox ID } \\
\text { et al. }\end{array}$ & 2000 & 32 & M & Coprocultivo & $\begin{array}{l}\text { Campylobacter } \\
\text { jejuni }\end{array}$ & $\begin{array}{l}\text { Diarrea, disnea, dolor } \\
\text { torácico }\end{array}$ & $\begin{array}{l}\text { Ritmo atrial bajo, } \\
\text { inversión simétrica de } \\
\text { onda T en V4 - V6, DI y } \\
\text { AVL }\end{array}$ & CPK elevada & No \\
\hline 14 & $\begin{array}{l}\text { Ma M, } \\
\text { et al. }\end{array}$ & 2007 & 23 & $\mathrm{~F}$ & $\begin{array}{l}\text { Biopsia de } \\
\text { miocardio }\end{array}$ & $\begin{array}{l}\text { Clostridium } \\
\text { novyi }\end{array}$ & $\begin{array}{l}\text { Dolor torácico, } \\
\text { escalofríos }\end{array}$ & $\begin{array}{l}\text { Bloqueo de rama } \\
\text { derecha, infradesnivel } \\
\text { ST difuso }\end{array}$ & CPK elevada & $\mathrm{Si}$ \\
\hline 15 & $\begin{array}{l}\text { Turpie DF } \\
\text { et al. }{ }^{34}\end{array}$ & 2009 & 45 & M & Coprocultivo & $\begin{array}{l}\text { Campylobacter } \\
\text { Jejuni }\end{array}$ & $\begin{array}{l}\text { Diarrea, emesis, } \\
\text { fiebre }\end{array}$ & $\begin{array}{l}\text { Fibrilación auricular con } \\
\text { elevación del ST en cara } \\
\text { anterolateral }\end{array}$ & $\begin{array}{l}\text { Troponina } \\
\text { elevada }\end{array}$ & No \\
\hline 16 & $\begin{array}{l}\text { Heinzl B } \\
\text { et al. }{ }^{35}\end{array}$ & 2010 & 17 & M & Coprocultivo & $\begin{array}{l}\text { Campylobacter } \\
\text { jejuni }\end{array}$ & $\begin{array}{l}\text { Fiebre, diarrea, dolor } \\
\text { torácico }\end{array}$ & $\begin{array}{l}\text { Elevación del ST en DI, } \\
\text { AVL, V4 y V5 }\end{array}$ & $\begin{array}{l}\text { Troponina } \\
\text { elevada }\end{array}$ & No \\
\hline 19 & $\begin{array}{l}\text { Fica A } \\
\text { et al. }{ }^{38}\end{array}$ & 2012 & 17 & M & $\begin{array}{l}\text { Cultivo para } \\
\text { Campylo- } \\
\text { bacter } \\
\text { (Medio } \\
\text { microaeró- } \\
\text { filo a T: } \\
42 C \text { ) }\end{array}$ & $\begin{array}{l}\text { Campylobacter } \\
\text { Jejuni }\end{array}$ & $\begin{array}{l}\text { Dolor abdominal, } \\
\text { diarrea, fiebre }\end{array}$ & $\begin{array}{l}\text { Elevación del ST en V1 - } \\
\text { V6 }\end{array}$ & CPK elevada & No \\
\hline
\end{tabular}




\begin{tabular}{|c|c|c|c|c|c|c|c|c|c|c|}
\hline No & Autor & Año & Edad & Sexo & $\begin{array}{l}\text { Método } \\
\text { diagnóstico }\end{array}$ & Microorganismo & Síntomas & Alteraciones en el EKG & Biomarcador & Muerte \\
\hline 20 & $\begin{array}{l}\text { De Cock D } \\
\text { et al. }\end{array}$ & 2012 & 34 & M & Coprocultivo & $\begin{array}{l}\text { Campylobacter } \\
\text { Jejuni }\end{array}$ & Diarrea, emesis & $\begin{array}{l}\text { Elevación del ST en V4 - } \\
\text { V6 }\end{array}$ & $\begin{array}{l}\text { Troponina } \\
\text { elevada }\end{array}$ & No \\
\hline 21 & $\begin{array}{l}\text { De Cock D } \\
\text { et al. }{ }^{39}\end{array}$ & 2012 & 21 & M & Coprocultivo & $\begin{array}{l}\text { Campylobacter } \\
\text { Jejuni }\end{array}$ & Diarrea, fiebre & $\begin{array}{l}\text { Elevación del ST en V4 - } \\
\text { V6 }\end{array}$ & CPK elevada & No \\
\hline 22 & $\begin{array}{l}\text { Brice J } \\
\text { et al. }{ }^{40}\end{array}$ & 2013 & 16 & M & Coprocultivo & $\begin{array}{l}\text { Salmonella } \\
\text { enteritidis }\end{array}$ & $\begin{array}{l}\text { Diarrea, fiebre, } \\
\text { escalofríos, dolor } \\
\text { torácico }\end{array}$ & $\begin{array}{l}\text { Bloqueo incompleto de } \\
\text { rama derecha }\end{array}$ & $\begin{array}{l}\text { Troponina } \\
\text { elevada }\end{array}$ & No \\
\hline 23 & $\begin{array}{l}\text { Panikkath R } \\
\text { et al. }{ }^{41}\end{array}$ & 2014 & 43 & M & ELISA & $\begin{array}{l}\text { Campylobacter } \\
\text { Jejuni }\end{array}$ & Diarrea & Ritmo sinusal, normal & $\begin{array}{l}\text { Troponina } \\
\text { elevada }\end{array}$ & No \\
\hline 24 & $\begin{array}{l}\text { Villablanca } \\
\text { P et al. }{ }^{15}\end{array}$ & 2015 & 19 & M & Coprocultivo & $\begin{array}{l}\text { Salmonella } \\
\text { berta }\end{array}$ & $\begin{array}{l}\text { Fiebre, escalofríos, } \\
\text { diarrea, dolor } \\
\text { abdominal, disnea, } \\
\text { dolor torácico }\end{array}$ & $\begin{array}{l}\text { Depresión del segmento } \\
\text { ST en V1, V2, elevación } \\
\text { en V5 y V6 }\end{array}$ & $\begin{array}{l}\text { Troponina } \\
\text { elevada }\end{array}$ & No \\
\hline 25 & $\begin{array}{l}\text { Gutiérrez de } \\
\text { la Varga } \\
\text { et al. }{ }^{42}\end{array}$ & 2017 & 55 & M & Coprocultivo & $\begin{array}{l}\text { Campylobacter } \\
\text { Jejuni }\end{array}$ & $\begin{array}{l}\text { Diarrea, dolor } \\
\text { abdominal, dolor } \\
\text { torácico }\end{array}$ & Alteración del ST difuso & $\begin{array}{l}\text { Troponina } \\
\text { elevada }\end{array}$ & No \\
\hline 26 & $\begin{array}{l}\text { Sundbom } \\
\text { et al. }{ }^{43}\end{array}$ & 2018 & 22 & M & Coprocultivo & $\begin{array}{l}\text { Salmonella } \\
\text { enteritidis }\end{array}$ & $\begin{array}{l}\text { Fiebre, dolor } \\
\text { abdominal, diarrea, } \\
\text { hematoquecia, dolor } \\
\text { torácico }\end{array}$ & $\begin{array}{l}\text { Elevación del ST en cara } \\
\text { anterior }\end{array}$ & $\begin{array}{l}\text { Troponina } \\
\text { elevada }\end{array}$ & No \\
\hline 27 & $\begin{array}{l}\text { Greefield } \\
\text { GM et al. }{ }^{44}\end{array}$ & 2018 & 31 & M & Coprocultivo & $\begin{array}{l}\text { Campylobacter } \\
\text { Jejuni }\end{array}$ & $\begin{array}{l}\text { Diarrea, fiebre, } \\
\text { mialgias, letargia, } \\
\text { dolor torácico. }\end{array}$ & Ritmo sinusal, normal & $\begin{array}{l}\text { Troponina } \\
\text { elevada }\end{array}$ & No \\
\hline
\end{tabular}


Tabla 3 Métodos imagenológicos

\begin{tabular}{|c|c|c|c|c|c|c|c|c|}
\hline No & Autor & Año & Edad & Sexo & Arteriografía & Ecocardiograma & $\begin{array}{l}\text { Resonancia } \\
\text { magnética }\end{array}$ & $\begin{array}{l}\text { Patología } \\
\text { con confir- } \\
\text { mación de } \\
\text { miocarditis }\end{array}$ \\
\hline 1 & $\begin{array}{l}\text { Hennigar } \\
\text { et al. }{ }^{21}\end{array}$ & 1953 & 2 & M & No & No & No & Sí \\
\hline 2 & $\begin{array}{l}\text { Rosenberg } \\
\text { et al. }{ }^{22}\end{array}$ & 1964 & 3 & $\mathrm{~F}$ & No & No & No & Sí \\
\hline 3 & Sanders et al. ${ }^{23}$ & 1964 & 62 & $\mathrm{~F}$ & No & No & No & Sí \\
\hline 4 & $\begin{array}{l}\text { Langaker } \\
\text { et al. } .^{24}\end{array}$ & 1973 & 76 & M & No & No & No & Sí \\
\hline 5 & $\begin{array}{l}\text { Simonsen } \\
\text { et al. }{ }^{25}\end{array}$ & 1980 & 24 & M & No & No & No & Sí \\
\hline 6 & Götz et al. ${ }^{26}$ & 1983 & 53 & M & No & No & No & Sí \\
\hline 7 & Burt et al. ${ }^{27}$ & 1990 & 29 & $M$ & No & No & No & Sí \\
\hline 8 & $\begin{array}{l}\text { Rubesteins JS } \\
\text { et al. }{ }^{28}\end{array}$ & 1993 & 8 & $\mathrm{~F}$ & No & $\begin{array}{l}\text { FEVI } 20 \% \text {, pobre } \\
\text { contractilidad del } \\
\text { ventrículo } \\
\text { izquierdo }\end{array}$ & No & Sí \\
\hline 9 & $\begin{array}{l}\text { Rubesteins JS } \\
\text { et al. }{ }^{28}\end{array}$ & 1993 & 10 & $\mathrm{~F}$ & No & $\begin{array}{l}\text { Trombo mural } \\
\text { biventricular }\end{array}$ & No & Sí \\
\hline 10 & León F et al. & 1997 & 59 & M & Normal & $\begin{array}{l}\text { FEVI } 30 \% \text {. } \\
\text { Hipoquinesia } \\
\text { generalizada } \\
\text { moderada en } \\
\text { todas las secciones } \\
\text { de las paredes de } \\
\text { los dos } \\
\text { ventrículos. } \\
\text { Control: } \\
\text { pericarditis aguda } \\
\text { con FE y diámetros } \\
\text { ventriculares } \\
\text { normales }\end{array}$ & No & Sí \\
\hline 11 & $\begin{array}{l}\text { Johnson JS } \\
\text { et al. }{ }^{29,30}\end{array}$ & 1998 & 83 & M & No & No & No & Sí \\
\hline 12 & $\begin{array}{l}\text { Neuwirth C, } \\
\text { et al. }{ }^{31}\end{array}$ & 1999 & 1 mes & M & No & No & No & Sí \\
\hline 13 & Cox ID et al. 32 & 2000 & 32 & M & No & $\begin{array}{l}\text { Ventrículo } \\
\text { izquierdo dilatado } \\
\text { con alteración } \\
\text { global de la } \\
\text { función sistólica. } \\
\text { Insuficiencia } \\
\text { mitral moderada }\end{array}$ & $\begin{array}{l}\text { Realce } \\
\text { tardío en } \\
\text { septum y } \\
\text { pared } \\
\text { lateral }\end{array}$ & No \\
\hline 14 & Ma M, et al. ${ }^{33}$ & 2007 & 23 & $\mathrm{~F}$ & No & $\begin{array}{l}\text { FEVI } 20 \% \text {, } \\
\text { Hipocinesia } \\
\text { ventricular } \\
\text { izquierda global }\end{array}$ & No & Sí \\
\hline 15 & $\begin{array}{l}\text { Turpie DF } \\
\text { et al. }{ }^{34}\end{array}$ & 2009 & 45 & M & $\begin{array}{l}\text { Normal. } \\
\text { Alteración } \\
\text { moderada } \\
\text { global de la } \\
\text { función } \\
\text { ventricular } \\
\text { izquierda }\end{array}$ & No & $\begin{array}{l}\text { Disminución } \\
\text { función } \\
\text { ventricular } \\
\text { sistólica, } \\
\text { realce } \\
\text { tardío sube- } \\
\text { picárdico en } \\
\text { pared ante- } \\
\text { roseptal }\end{array}$ & No \\
\hline
\end{tabular}


Tabla 3 (continuación)

\begin{tabular}{|c|c|c|c|c|c|c|c|c|}
\hline No & Autor & Año & Edad & Sexo & Arteriografía & Ecocardiograma & $\begin{array}{l}\text { Resonancia } \\
\text { magnética }\end{array}$ & $\begin{array}{l}\text { Patología } \\
\text { con confir- } \\
\text { mación de } \\
\text { miocarditis }\end{array}$ \\
\hline 16 & $\begin{array}{l}\text { Hibbert B } \\
\text { et al. }{ }^{36}\end{array}$ & 2010 & 25 & M & Normal & No & $\begin{array}{l}\text { Realce } \\
\text { tardío de } \\
\text { distribución } \\
\text { miocárdica } \\
\text { y subendo- } \\
\text { cárdica }\end{array}$ & No \\
\hline 17 & $\begin{array}{l}\text { Kratzer C } \\
\text { et al. }{ }^{37}\end{array}$ & 2010 & 19 & M & $\begin{array}{l}\text { Normal. } \\
\text { Ventriculo- } \\
\text { grama: Área } \\
\text { hipoquiné- } \\
\text { tica en la } \\
\text { región del } \\
\text { ápex } \\
\text { compatible } \\
\text { con } \\
\text { miocarditis }\end{array}$ & $\begin{array}{l}\text { FEVI normal, } \\
\text { normal }\end{array}$ & $\begin{array}{l}\text { Realce } \\
\text { tardío } \\
\text { difuso en } \\
\text { ventrículo } \\
\text { izquierdo } \\
\text { hasta tercio } \\
\text { medial }\end{array}$ & No \\
\hline 18 & $\begin{array}{l}\text { Heinzl B, } \\
\text { et al. }{ }^{35}\end{array}$ & 2010 & 17 & M & No & $\begin{array}{l}\text { FEVI normal, } \\
\text { normal }\end{array}$ & $\begin{array}{l}\text { Realce } \\
\text { tardío en } \\
\text { pared ante- } \\
\text { roinferior }\end{array}$ & No \\
\hline 19 & Fica A et al. ${ }^{38}$ & 2012 & 17 & M & No & Normal & $\begin{array}{l}\text { Realce } \\
\text { segmentario } \\
\text { focal del } \\
\text { miocardio } \\
\text { de } \\
\text { ventrículo } \\
\text { izquierdo }\end{array}$ & No \\
\hline 20 & $\begin{array}{l}\text { De Cock D } \\
\text { et al. }{ }^{39}\end{array}$ & 2012 & 34 & M & $\begin{array}{l}\text { Normal } \\
\text { Ventriculo- } \\
\text { grama: FEVI } \\
40 \%, \\
\text { hipoquinesia } \\
\text { difusa }\end{array}$ & No & $\begin{array}{l}\text { Realce } \\
\text { tardío } \\
\text { confinado a } \\
\text { subepicar- } \\
\text { dio de pared } \\
\text { inferolateral }\end{array}$ & No \\
\hline 21 & $\begin{array}{l}\text { De Cock D } \\
\text { et al. }{ }^{39}\end{array}$ & 2012 & 21 & M & No & FEVI 40\%, normal & $\begin{array}{l}\text { Edema de } \\
\text { pared } \\
\text { lateral del } \\
\text { ventrículo } \\
\text { izquierdo }\end{array}$ & No \\
\hline 22 & Brice $\mathrm{J}$ et al. ${ }^{40}$ & 2013 & 16 & M & No & Normal, FEVI: 68\% & $\begin{array}{l}\text { Realce } \\
\text { ínferobasal } \\
\text { nodular } \\
\text { intramiocár- } \\
\text { dico }\end{array}$ & No \\
\hline 23 & $\begin{array}{l}\text { Panikkath R } \\
\text { et al. }{ }^{41}\end{array}$ & 2014 & 43 & M & Normal & $\begin{array}{l}\text { FEVI } 68 \% \\
\text { Anormalidades } \\
\text { segmentarias de la } \\
\text { contractibilidad } \\
\text { en la porcion } \\
\text { inferior del } \\
\text { septum }\end{array}$ & $\begin{array}{l}\text { Realce } \\
\text { epicárdico y } \\
\text { medio } \\
\text { miocárdico } \\
\text { en pared } \\
\text { anterolate- } \\
\text { ral y septum } \\
\text { interventri- } \\
\text { cular }\end{array}$ & No \\
\hline
\end{tabular}




\begin{tabular}{|c|c|c|c|c|c|c|c|c|}
\hline No & Autor & Año & Edad & Sexo & Arteriografía & Ecocardiograma & $\begin{array}{l}\text { Resonancia } \\
\text { magnética }\end{array}$ & $\begin{array}{l}\text { Patología } \\
\text { con confir- } \\
\text { mación de } \\
\text { miocarditis }\end{array}$ \\
\hline 24 & $\begin{array}{l}\text { Villablanca P } \\
\text { et al. }{ }^{15}\end{array}$ & 2015 & 19 & $M$ & No & $\begin{array}{l}\text { Disminución de } \\
\text { FEVI a } 40 \%\end{array}$ & $\begin{array}{l}\text { Captación } \\
\text { subepicár- } \\
\text { dica y en } \\
\text { miocardio } \\
\text { en región } \\
\text { posterior, } \\
\text { inferior, } \\
\text { anterior }\end{array}$ & No \\
\hline 25 & $\begin{array}{l}\text { Gutiérrez de la } \\
\text { Varga et al. }{ }^{42}\end{array}$ & 2017 & 55 & M & No & $\begin{array}{l}\text { FEVI normal, } \\
\text { normal }\end{array}$ & $\begin{array}{l}\text { Edema, sin } \\
\text { realce } \\
\text { tardío }\end{array}$ & No \\
\hline 26 & $\begin{array}{l}\text { Sundbom } \\
\text { et al. }{ }^{43}\end{array}$ & 2018 & 22 & M & No & $\begin{array}{l}\text { Disminución de } \\
\text { FEVI a } 46 \%\end{array}$ & $\begin{array}{l}\text { Edema e } \\
\text { inflamación } \\
\text { en área } \\
\text { inferior e } \\
\text { inferolateral }\end{array}$ & No \\
\hline 27 & $\begin{array}{l}\text { Greefield GM } \\
\text { et al. }{ }^{44}\end{array}$ & 2018 & 31 & M & No & $\begin{array}{l}\text { FEVI normal, } \\
\text { función diastólica } \\
\text { normal } \\
\text { Normal }\end{array}$ & $\begin{array}{l}\text { Edema } \\
\text { miocárdico } \\
\text { localizado y } \\
\text { realce en } \\
\text { pared } \\
\text { inferolateral } \\
\text { y basal }\end{array}$ & No \\
\hline
\end{tabular}
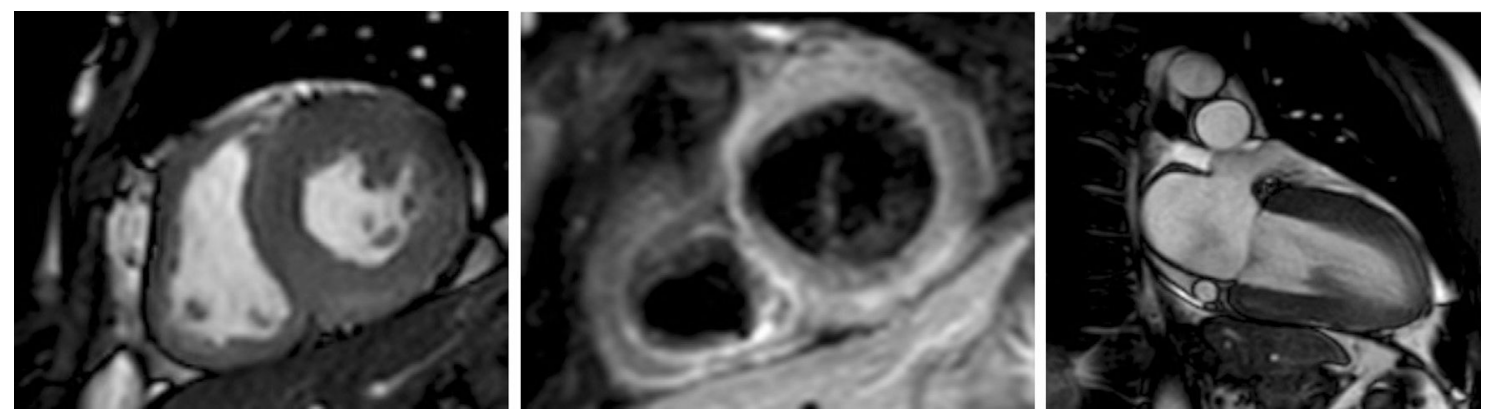

Figura 1 Resonancia magnética cardíaca; en la caracterización tisular se observa edema lineal en localización subepicárdica en el segmento inferior e inferolateral. En correlación con esta zona de edema, se observa realce tardío.

\section{Pregunta de investigación}

¿Cuáles son las características clínicas de los pacientes con miocarditis asociada a infección por bacterias gram negativas?

\section{Metodología}

Se realizó una revisión sistemática de la literatura con el objetivo de identificar los casos de miocarditis causadas por bacilos gram negativos. La población objetivo de estudio fueron los pacientes (tanto población pediátrica como adultos) con diagnóstico de infección bacteriana por bacilo gram negativo mediante cultivo, técnicas moleculares o histopatología, y quienes tenían biopsia de miocardio o, en su defecto, resonancia magnética cardiaca con hallazgos sugestivos de miocarditis, con el fin de excluir posibles casos de miocardiopatía por sepsis que pudiera generar alteraciones en el electrocardiograma o ecocardiograma sin tratarse de miocarditis.

Se emplearon las bases de datos: Pubmed, Ovid, Scopus, SciELO y LILACS sin exclusión por tipo de idioma. Se incluyeron reportes de casos, editoriales y cohortes. Se utilizaron los términos MESH: ("Myocarditis"'[Mesh]) OR Pancarditis AND "Enterobacteriaceae"'[Mesh], ("Myocarditis"'[Mesh]) OR Pancarditis AND "Salmonella"'[Mesh], ("Myocarditis"'[Mesh]) OR Pancarditis AND "Campylobacter"'[Mesh], ("Myocarditis"'[Mesh]) OR Pancarditis AND "Shigella"'[Mesh], ("Myocarditis"'[Mesh]) OR 
Pancarditis AND "Cholera"'[Mesh], ("Myocarditis"'[Mesh]) OR Pancarditis AND "Vibrio"'[Mesh], ("Myocarditis"'[Mesh]) OR Pancarditis AND "Typhoid fever"'[Mesh], ("Myocarditis"'[Mesh]) OR Pancarditis AND "Yersinia"'[Mesh] / ("Myocarditis"'[Mesh]) OR Pancarditis AND "Yersinia infections"'[Mesh), ("Myocarditis"'[Mesh]) OR Pancarditis AND "Clostridium"'[Mesh].

Con esta búsqueda se obtuvieron 742 artículos, de los cuales 352 se encontraban repetidos. De los 390 artículos restantes se excluyeron 366 y se incluyeron 24 estudios en modalidad de reporte de caso que cumplieron la definición de miocarditis por biopsia endomiocárdica o hallazgos en resonancia magnética.

\section{Resultados}

Se incluyeron 24 artículos en los cuales se reportaron 27 pacientes que cumplían el diagnóstico de miocarditis mediante estudio con resonancia magnética o biopsia de endomiocardio. Para confirmar el diagnóstico, en 14 de los casos se realizó resonancia magnética y en 13 se hizo biopsia.

El $81 \%$ eran de sexo masculino, con edades entre 1 mes a 83 años, 9 de ellos con edades menores a 18 años. El promedio de edad fue de 31 años. El $37 \%$ de los pacientes falleció (10 pacientes).

El síntoma principal fue diarrea en $80 \%$ de los casos $(21$ pacientes), seguido de fiebre 53\% (14 pacientes), dolor torácico 38\% (10 pacientes), vómito 19\% (5 pacientes) y disnea $15 \%$ (4 pacientes). En uno de los casos no hubo reporte de sintomatología.

Se encontró reporte de electrocardiograma en 22 de los casos; el hallazgo más común fue elevación del segmento ST en 8 pacientes $(36,3 \%)$. Se encontró alteración en la onda T (inversión o aplanamiento) en 4 pacientes $(18,1 \%)$, seguido de infradesnivel del segmento ST (3 pacientes, 13,6\%).

Al menos un biomarcador fue reportado en 15 de los pacientes, de los cuales 5 correspondían a CPK (creatina fosfoquinasa) y 10 a troponina, con elevación de los mismos. En 16 de los casos se realizó ecocardiograma, el cual fue normal en 5 de estos (31,2\%); en $50 \%$ ( 8 casos) se reportó disminución de la fracción de eyección y en $25 \%$ (4 casos) hipoquinesia difusa.

El microorganismo más común fue Campylobacter jejuni, el cual se aisló en 10 de los pacientes (70\% de los casos); como género, Salmonella $s p$ fue la etiología en 11 de los casos, de los cuales ninguno fue Salmonella typhi. De los 27 casos, en 5 se encontró bacteriemia asociada (18,5\%).

\section{Discusión}

La etiología bacteriana en el curso de miocarditis es una entidad infrecuente teniendo en cuenta el bajo número de casos encontrados en la literatura. Entre las enterobacterias causales se encuentra Salmonella spp, microorganismo que puede causar complicaciones como endocarditis, así como infección de aneurismas, miocarditis y pericarditis ${ }^{14}$.

En la revisión sistemática llevada a cabo por Villablanca et al. ${ }^{15}$ sobre miocarditis por Salmonella spp se incluían artículos con pacientes que tuvieran evidencia clínica, electrocardiográfica o por imágenes de compromiso miocárdico, por lo que al no limitarlos a hallazgos de biopsia endomiocárdica o resonancia magnética el total de casos de miocarditis secundario a infección por Salmonella spp asciende a 24 casos. En esta revisión también se encontró un predominio masculino en la edad adulta, con edad media de presentación en la cuarta década, similar a nuestros resultados.

Las formas invasivas de la enfermedad están dadas por fiebre entérica o bacteriemia por Salmonella no tifoidea ${ }^{16}$. La presentación clínica es variable ya que puede comprometer varios sistemas. En el caso de los pacientes que cursan con miocarditis, Villablanca et al. ${ }^{15}$ encontraron que la fiebre se presentaba en $66,6 \%$ de los casos; sin embargo, menos del $25 \%$ presentaba diarrea. En nuestra revisión, al aislar a los pacientes que tenían infección por Salmonella spp (11 pacientes), 10 de ellos presentaron diarrea como manifestación clínica.

Los hallazgos electrocardiográficos son similares a los del estudio mencionado ${ }^{15}$ con predominio de alteración del segmento ST; así mismo, en las alteraciones ecocardiográficas se encontró con mayor frecuencia disminución de la fracción de eyección y alteraciones en la contractilidad.

La disfunción miocárdica inducida por sepsis comparte hallazgos ecocardiográficos, elevación de biomarcadores y trastornos del ritmo con la miocarditis; en la miocardiopatía por sepsis también puede encontrarse disminución en la fracción de eyección, alteración segmentaria en la contractilidad y disfunción diastólica ${ }^{17,18}$.

Según los autores y con base en la búsqueda realizada, no hay estudios que describan las características en resonancia magnética de la miocardiopatía por sepsis; el uso de la misma en este contexto se reportó en un caso para establecer el diagnóstico de miocarditis en una paciente con sepsis de origen urinario ${ }^{19}$. De hecho, esta entidad aún no cuenta con criterios diagnósticos, lo que limita establecer su verdadera prevalencia. Los criterios propuestos al momento. incluyen la presencia de un síndrome de falla cardíaca no relacionado con isquemia y asociado a sepsis, acompañado de dilatación ventricular izquierda con presión o baja presión de llenado, alteración en contractilidad y disfunción ventricular derecha o izquierda en ausencia de respuesta a infusión de volumen ${ }^{20}$. Los mecanismos que producen la disfunción miocárdica en sepsis incluyen alteración en canales de calcio, disfunción mitocondrial, circulación de citoquinas inflamatorias y patrones moleculares asociados a daño ${ }^{20}$.

Por lo anterior, en el contexto de sepsis de origen gastrointestinal o con sospecha de infección por Enterobacterias y disfunción miocárdica se propone el uso de resonancia cardíaca o biopsia endomiocárdica en caso de sospecha de miocarditis que esté asociada a falla cardíaca congestiva severa, disfunción ventricular severa, arritmias que amenacen la vida o en el contexto de un síndrome clínico de disfunción miocárdica refractario al tratamiento y habiendo excluido otras etiologías ${ }^{1}$.

\section{Conclusión}

La infección por Salmonella spp es una enfermedad prevalente en los países en desarrollo, sin cifras establecidas aún en Colombia. Entre las complicaciones durante la infección gastrointestinal se incluye la inflamación miocárdica, la cual 
debe sospecharse en pacientes con síntomas cardiovasculares.

\section{Conflicto de intereses}

Los autores no tienen actividades o relaciones con terceros que hayan influido en la publicación de este artículo ni en las conclusiones presentadas.

\section{Anexo. Material adicional}

Se puede consultar material adicional a este artículo en su versión electrónica disponible en doi:10.1016/j.rccar.2020.05.006.

\section{Bibliografía}

1. Sinagra G, Anzini M, Pereira NL, Bussani R, Finocchiaro G, Bartunek J, et al. Myocarditis in clinical practice. Mayo Clin Proc. 2016;91:1256-66.

2. Felker GM, Thompson RE, Hare JM, Hruban RH, Clemetson DE, Howard DL, et al. Underlying causes and long-term survival in patients with initially unexplained cardiomyopathy. N Engl J Med. 2000;342:1077-84.

3. Dennert R, Crijns HJ, Heymans S. Acute viral myocarditis. Eur Heart J. 2008;29:2073-82.

4. Massilamany C, Huber SA, Cunningham MW, Reddy J. Relevance of molecular mimicry in the mediation of infectious myocarditis. J Cardiovasc Transl Res. 2014;7:165-71.

5. Maisch B, Pankuweit S. Standard and etiology-directed evidence-based therapies in myocarditis: state of the art and future perspectives. Heart Fail Rev. 2013;18:761-95.

6. Haddad F, Berry G, Doyle RL, Martineau P, Leung T-K, Racine N. Active bacterial myocarditis: a case report and review of the literature. J Heart Lung Transplant. 2007;26:745-9.

7. Wasi F, Shuter J. Primary bacterial infection of the myocardium. Front Biosci. 2003;8:s228-31.

8. Berry GJAK. Pathology of human myocarditis. In: Myocarditis: from bench to bedside. 2003:325-70.

9. Friedrich MG, Sechtem U, Schulz-Menger J, Holmvang G, Alakija P, Cooper LT, et al. Cardiovascular magnetic resonance in myocarditis: A JACC White Paper. J Am Coll Cardiol. 2009;53:1475-87.

10. Haaf P, Buser PT. Map to the future of cardiac magnetic resonance in myocarditis. Eur Heart $\mathrm{J}$ Cardiovasc Imag. 2017; 18:752-3.

11. Mahrholdt H, Goedecke C, Wagner A, Meinhardt G, Athanasiadis $A$, Vogelsberg $\mathrm{H}$, et al. Cardiovascular magnetic resonance assessment of human myocarditis: a comparison to histology and molecular pathology. Circulation. 2004;109: 1250-8.

12. Fung G, Luo H, Qiu Y, Yang D, McManus B. Myocarditis. Circ Res. 2016;118:496-514.

13. Caforio ALP, Pankuweit S, Arbustini E, Basso C, Gimeno-Blanes $\mathrm{J}$, Felix SB, et al. Current state of knowledge on aetiology, diagnosis, management, and therapy of myocarditis: A position statement of the European Society of Cardiology Working Group on Myocardial and Pericardial Diseases. Eur Heart J. 2013;34:2636-48.

14. Hibbert B, Costiniuk C, Hibbert R, Joseph P, Alanazi H, Simard T, et al. Cardiovascular complications of Salmonella enteritidis infection. 2010;26:323-5.

15. Villablanca P, Mohananey D, Meier G, Yap JE, Chouksey S, Abegunde AT. Salmonella berta myocarditis: Case report and systematic review of non-typhoid Salmonella myocarditis. World J Cardiol. 2015;7:931-7.

16. Franco-Paredes C, Khan MI, Gonzalez-Diaz E, Santos-Preciado JI, Rodriguez-Morales AJ, Gotuzzo E. Enteric fever: a slow response to an old plague. PLoS Negl Trop Dis. 2016;10:e0004597.

17. Walley KR. Sepsis-induced myocardial dysfunction. Curr Opin Crit Care. 2018;24:292-9.

18. Narvaez I, Canabal A, Martin C, Sanchez M, Moron A, Alcala $\mathrm{J}$, et al. Incidence and evolution of sepsis-induced cardiomyopathy in a cohort of patients with sepsis and septic shock. Med Intensiva. 2018;42:283-91.

19. De Lazzari M, Marra MP, Cacciavillani L, Cucchini U, Rossi S, Iliceto $\mathrm{S}$, et al. Inside myocardial dysfunction in septic shock: mechanism of troponin release highlighted by cardiac magnetic resonance. J Cardiovasc Med (Hagerstown). 2017;18:818-9.

20. Martin L, Derwall M, Al Zoubi S, Zechendorf E, Reuter DA, Thiemermann C, et al. The Septic Heart: Current Understanding of Molecular Mechanisms and Clinical Implications. Chest. 2019;155:427-37.

21. Hennigar GR, Thabet R, Bundy WE, Sutton LE. Salmonellosis complicated by pancarditis: report of a case with autopsy findings. J Pediatr. 1953;43:524-31.

22. Rosenberg HS, Mcnamara DG. Acute myocarditis in infancy and childhood. Prog Cardiovasc Dis. 1964;7:179-97.

23. Sanders V, Misanik LM. Salmonella myocarditis: Report of a case with ventricular rupture. Am Heart J. 2005;68:914-6.

24. Langaker OM, Svanes K. Myocardial abscess due to Salmonella typhimurium. Br Heart J. 1973;35:871-3.

25. Simonsen J, Falk E. A case of sudden cardiac death in connection with Salmonella typhimurium infection. Forensic Sci Int. 1980;16:283-7.

26. Gotz M, Juchems R. Myocarditis caused by Salmonella typhimurium. Klin Wochenschr. 1983;61:1153-7.

27. Burt CR, Proudfoot JC, Roberts M, Horowitz RH. Fatal myocarditis secondary to salmonella septicemia in a young adult. J Emerg Med. 1990;8:295-7.

28. Rubenstein JS, Noah ZL, Zales VR, Shulman ST. Acute myocarditis associated with Shigella sonnei gastroenteritis. J Pediatr. 1993; 122:82-4

29. Leon F, Badui E, Campos A, Enciso R, Fakih T, Guadarrama $M$, et al. Cholera and myocarditis-a case report. Angiology. 1997;48:545-9.

30. Johnson JS, Croall J, Power JS, Armstrong GR. Fatal Serratia marcescens meningitis and myocarditis in a patient with an indwelling urinary catheter. J Clin Pathol. 1998;51:789-90.

31. Neuwirth C, Francois C, Laurent N, Pechinot A. Myocarditis due to Salmonella virchow and sudden infant death. Lancet. 1999;354:1004.

32. Cox ID, Fluck DS, Joy MD. Campylobacter myocarditis; loose bowels and a baggy heart. Eur J Heart Fail. 2001;3:105-7.

33. Ma M, Boyd JT, Trinh HT, Coombs JW, Fermann GJ. Fatal myocarditis due to Clostridium novyi type $B$ in a previously healthy woman: case report and literature review. Scand J Infect Dis. 2007;39:77-80.

34. Turpie DFS, Forbes KJ, Hannah A, Metcalfe MJ, McKenzie H, Small GR. Food-the way to a man's heart: a mini-case series of Campylobacter perimyocarditis. Scand J Infect Dis. 2009;41(6-7):528-31.

35. Heinzl B, Kostenberger M, Nagel B, Sorantin E, Beitzke A, Gamillscheg A. Campylobacter jejuni infection associated with myopericarditis in adolescents: report of two cases. Eur J Pediatr. 2010;169:63-5.

36. Hibbert B, Costiniuk C, Hibbert R, Joseph P, Alanazi H, Simard $\mathrm{T}$, et al. Cardiovascular complications of Salmonella enteritidis infection. Can J Cardiol [Internet]. 2010;26:e323-5. Disponible en: http://linkinghub.elsevier.com/ retrieve/pii/S0828282X1070444X. 
37. Kratzer C, Wolf F, Graninger W, Weissel M. Acute cardiac disease in a young patient with Campylobacter jejuni infection: a case report. Wien Klin Wochenschr. 2010;122(9-10):315-9.

38. Fica A, Seelmann D, Porte L, Eugenin D, Gallardo R. A case of myopericarditis associated to Campylobacter jejuni infection in the Southern Hemisphere. Brazilian J Infect Dis [Internet]. 2012;16:294-6. Disponible en: https://doi.org/10.1016/S14138670(12)70327-6.

39. De Cock D, Hiltrop N, Timmermans P, Dymarkowski S, Van Cleemput J. Myocarditis associated with Campylobacter enteritis: report of three cases. Circ Heart Fail. 2012;5:e19-21.

40. Brice J, Baumard S, Loeb F, Brasme L, Jaussaud R, N'Guyen Y. Salmonella enteritidis infection complicated by acute myocarditis. Med Mal Infect. 2013;43:248-50.
41. Panikkath R, Costilla V, Hoang P, Wood J, Gruden JF, Dietrich B, et al. Chest pain and diarrhea: a case of Campylobacter jejuniassociated myocarditis. J Emerg Med. 2014;46:180-3.

42. Gutierrez de la Varga L, Rodriguez Suarez ML, Corros Vicente C. Myocarditis associated with Campylobacter jejuni infection. Medicina Cílnica. 2017;148:333-4.

43. Sundbom P, Suutari A-M, Abdulhadi K, Broda W, Csegedi M. Salmonella enteritidis causing myocarditis in a previously healthy 22-year-old male. Oxford Medical Case Reports. England; 2018:106.

44. Greenfield GM, Mailey J, Lyons K, Trouton TG. Acute myocarditis secondary to acute Campylobacter jejuni infection. Clin Med. 2018;18:98-9. 